of a device and control the distribution of the two GST phases in the plane based on its performance. Such a scheme would reduce the need for time-consuming 'blind' fabrication runs as well as for large-scale numerical simulations in the design process of complex graded metasurfaces. Ultimately, the approach of Taubner and collaborators offers the potential for defining flat midinfrared optical devices (such as lenses, beam shapers and holograms) on demand, which can be dynamically adjusted to changing requirements in a similar manner as data can be stored and erased on a rewritable compact disc.

While the approach unlocks a plethora of exciting opportunities, it currently faces multiple technical limitations, the most important of which is the rather small number of cycles in which the GST phase can be switched before degradation occurs. However, it can be expected that further optimization of the system can mitigate this limitation. Several fundamental limitations also apply to SPhP-based systems, including the maximum electric field enhancement that can be achieved ${ }^{10}$. Last but not least, since SPhPs can only exist inside the rather limited spectral range of the Reststrahlen band in the mid-infrared, demonstration of this concept with plasmonic or Mie-resonant dielectric systems would be crucial to extend the possibility for rewritable subwavelength optics to near-infrared and visible frequencies.

\section{Isabelle Staude is at the Institute of} Applied Physics, Abbe Center of Photonics,
Friedrich-Schiller-Universität Jena, 07743 Jena, Germany. Carsten Rockstuhl is at the Institute of Theoretical Solid State Physics, Karlsruhe Institute of Technology, 76131 Karlsruhe, Germany and at the Institute of Nanotechnology, Karlsruhe Institute of Technology, 76021 Karlsruhe, Germany. e-mail: isabelle.staude@uni-jena.de; carsten.rockstuhl@kit.edu

\section{References}

1. MacDonald, K. F. \& Zheludev, N. I. Laser Photon. Rev. 4, 562-567 (2010).

2. Kamaraju, N. et al. Light: Sci. Appl. 3, e155 (2014)

3. Li, P. et al. Nature Mater. 15, 870-875 (2016).

4. Caldwell, J. et al. Nanophotonics 4, 44-68 (2015).

5. Dai, S. et al. Science 343, 1125-1129 (2014).

6. Sámson, Z. L. et al. Appl. Phys. Lett. 96, 143105 (2010)

7. Tittl, A. et al. Adv. Mater. 27, 4597-4603 (2015)

8. Ríos, C. et al. Nature Photon. 9, 725-732 (2015).

9. Yu, N. \& Capasso, F. Nature Mater. 13, 139-150 (2014)

10. Khurgin, J. B. Nature Nanotech. 10, 2-6 (2015).

\title{
A NEW TWIST FOR OLEDs
}

Ever since organic light-emitting diodes (OLEDs) were invented in the 1980s, they have faced a problem which limits the efficiency of converting electrical energy to light emission. In these devices, some of the first of which used conjugated polymers as the emitters, photons are emitted owing to the recombination of an electron and a hole injected into the material from electrodes. These charge carriers experience mutual attraction and form a paired excitation called an exciton that may eventually decay by light emission. But there are four possible combinations of the electron and hole spins: one in which they are antiparallel (a singlet state) and three with the spins parallel (a triplet state). And here's the rub: only the singlet, in usual circumstances, is emissive. The triplet states eventually decay thermally, squandering their energy and limiting the 'internal' efficiency of the OLED devices - the efficiency of emission for each exciton created - to $25 \%$ at best.

How can the remaining $75 \%$ of this energy be harnessed? Several tricks have been tried. One of the most effective is to find materials for which the triplet states can be emissive too. Many OLEDs now use small organic and organometallic molecules instead of polymers (as indeed did the very first of them), and certain organometallic complexes of heavy metal atoms such as platinum and iridium can possess emissive triplet states if the light is produced by phosphorescence rather than fluorescence (Baldo, M. A. et al. Nature 395, 151-154; 1998). What's more, the process called intersystem crossing (ISC), by which singlet and triplet states are interconverted, is fast in these phosphorescent OLEDs and allows the emissive triplet states to harvest the energy of singlet states too.

That's one way to harness the energy. However, it's hard to make stable blue-light emitters for colour screens from these materials. Now Di et al. describe another way (Di, D. et al. Preprint at http://www. arxiv.org/abs/1606.08868; 2016). The authors enhanced the rate of ISC in a class of gold- and copper-containing organometallic compounds to enable triplet excitons to become emissive singlets. The trick comes down to shrinking to zero the energy gap between the two types of spin state, at which point they can interconvert most easily.

These compounds have a propeller shape in which two roughly planar organic groups, containing multiple ring structures, are attached above and below a central metal atom. These two groups can pivot around the metal, and the energy of the complex depends on their relative orientation, measured by the dihedral angle between the propeller 'blades'. For a triplet state, the

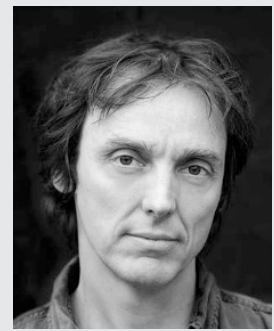

PHILIP BALL

energy increases slightly as this angle varies from zero (parallel) to $90^{\circ}$; for a singlet, there is an opposite and more marked variation, the $90^{\circ}$ orientation being energetically favourable. So there's an intermediate orientation where the energies are the same and ISC is facile.

In principle, this allows for all of the triplet energy to be harvested as singlet emission: an internal efficiency of $100 \%$. The overall efficiency of electrical-to-light energy conversion will be considerably smaller, since there are other sources of energy loss. But Di et al. show that they can make devices from solution-processed thin films of these materials with efficiencies of up to $27 \%$, which compares well with state-of-the-art OLEDs made by more demanding high-vacuum processing. It's a piece of clever molecular quantum engineering that could even end up coupling electrical and optical behaviour with mechanical motion. 